\title{
Proton and Deuteron Spin Structure Function Measurements in the Resonance Region
}

\author{
Frank R. Wesselmann \\ University of Virginia, Charlottesville, Virginia 22904 \\ For the RSS Collaboration
}

\begin{abstract}
The RSS collaboration has measured the spin structure functions of the proton and the deuteron at Jefferson Lab using the Hall C HMS spectrometer, a polarized electron beam and a polarized solid target. The asymmetries $A_{\|}$and $A_{\perp}$ were measured in the region of the nucleon resonances $(0.82 \mathrm{GeV}<W<1.98 \mathrm{GeV})$ at an average four momentum transfer of $Q^{2}=1.3 \mathrm{GeV}^{2}$. The extracted spin structure functions and their kinematic dependence will make a significant contribution in the study of higher-twist effects and polarized duality tests. A description of the experiment and the latest findings of the analysis will be presented.
\end{abstract}

\section{INTRODUCTION}

Spin structure functions of the nucleon have been measured since the late 1970's, for example at SLAC [1], CERN [2], and DESY [3]. Most of the experimental studies have concentrated on the high $Q^{2}$ region, where perturbative QCD works well with electrons scattering from an essentially free constituent. The low and intermediate energy range (up to $6 \mathrm{GeV}$ ) polarized electron beam at the Thomas Jefferson National Accelerator Facility (TJNAF) gives us a unique opportunity to study the nucleon spin structure in the lower energy regime where quarks are not asymptotically free and pQCD does not apply. Measuring the spin structure in this $Q^{2}$ region will allow us to study the contribution of individual nucleon resonances to the spin structure function.

One of the measurable quantities in polarized electron-nucleon scattering is the cross section asymmetry, simply a normalized difference between the scattering cross sections with the electron and nucleon spins aligned parallel and anti-parallel (or $\pm 90^{\circ}$ in the transverse case). Experiment E01-006 at Jefferson Laboratory has measured the asymmetries $A_{\|}$and $A_{\perp}$ at $Q^{2} \approx 1.3 \mathrm{GeV}^{2}$ from proton and deuteron targets. We define these asymmetries as:

$$
A_{\|}=\frac{d \sigma^{\downarrow \uparrow}-d \sigma^{\uparrow \uparrow}}{d \sigma^{\downarrow \uparrow}+d \sigma^{\uparrow \uparrow}}
$$

and

$$
A_{\perp}=\frac{d \sigma^{\downarrow \rightarrow}-d \sigma^{\uparrow \rightarrow}}{d \sigma^{\downarrow \rightarrow}+d \sigma^{\uparrow \rightarrow}}
$$

This high precision measurement of both $A_{\|}$and $A_{\perp}$ will allow us to extract the virtual photon asymmetries $A_{1}\left(v, Q^{2}\right)$ and $A_{2}\left(v, Q^{2}\right)$ without using any assumption or models. $A_{1}$ and $A_{2}$ provide a description of the quark contribution to the nucleon spin.

CP675, Spin 2002: 15th Int'l. Spin Physics Symposium and Workshop on Polarized Electron

Sources and Polarimeters, edited by Y. I. Makdisi, A. U. Luccio, and W. W. MacKay

(C) 2003 American Institute of Physics 0-7354-0136-5/03/\$20.00

606 
The nucleon spin structure in the resonance region as well as the connection to the DIS measurements can be studied. Also, the effects of quark-gluon interactions, twist-3 terms, and local polarized duality can be explored from this measurement.

\section{EXPERIMENT}

The experiment was conducted at Jefferson Lab in Hall $\mathrm{C}$ during two months in early 2002 using a continuous, polarized electron beam with energy of $5.7 \mathrm{GeV}$. The beam polarization was measured by a Moller polarimeter installed upstream of the target; the average beam polarization was about $70 \%$. The beam helicity was flipped at $30 \mathrm{~Hz}$ on a pseudo-random basis. To minimize any false asymmetry or bias, we determined the beam charge asymmetry over five minutes intervals and the measured value was fed back to the helicity control device at the injector.

Frozen ${ }^{15} \mathrm{NH}_{3}$ and ${ }^{15} \mathrm{ND}_{3}$ were used as material for proton and deuteron targets, respectively. The target polarization was achieved via a Dynamic Nuclear Polarization technique and measured by an NMR system using pickup coils embedded in the target material. The average target polarization was around $80 \%$ for ${ }^{15} \mathrm{NH}_{3}$, and for ${ }^{15} \mathrm{ND}_{3}$ around $20 \%$. For the $A_{\perp}$ measurement, the whole target was rotated by $90^{\circ}$ from its parallel position.

Scattered electrons were detected using the High Momentum Spectrometer (HMS), positioned at a scattering angle of $13.15^{\circ}$. Two different HMS momentum setting were used to cover wider kinematic range, as shown in figure 1, resulting in an average $Q^{2}$ of $\approx 1.3 \mathrm{GeV}^{2}$. A detector package consisting of hodoscope planes, wire chambers, a Cerenkov counter and a lead glass calorimeter allowed for particle identification and measurement of the event kinematics.



FIGURE 1. Statistical and Kinematic Coverage of RSS Experiment. 


\section{RESULTS}

Approximately 160 million scattering events were recorded on the proton target, and 350 million on deuteron. Data analysis is currently underway, including offline calibration of the spectrometer optics, tuning of tracking and particle identification and extracting beam and target polarization. The results from the first pass analysis of the count asymmetries, shown in figure 2, are very promising. These preliminary asymmetries have been extracted with online values for the beam and target polarizations, which will later be replaced with offline numbers. The target dilution factor and radiative corrections were not applied to the plotted data.
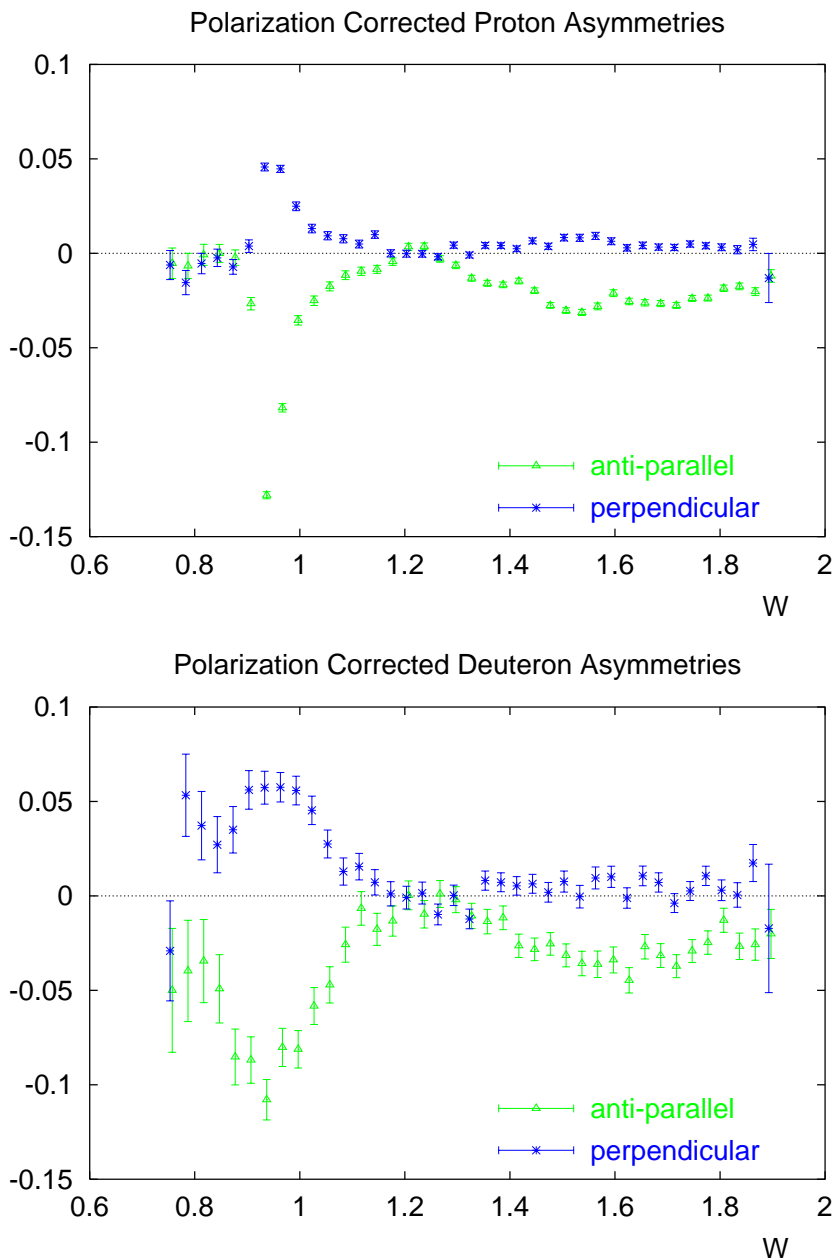

FIGURE 2. Preliminary result for $\mathrm{NH}_{3}$ and $\mathrm{ND}_{3}$ asymmetries, with beam-target polarization parallel and perpendicular. The experiment's anti-parallel alignment of target polarization and beam direction results in the negative sign for $A_{\|}$. 


\section{OUTLOOK}

One of main physics goals of the program is to study the $W$ dependence of the transverse and longitudinal polarized structure functions of the proton and deuteron in the resonance region. This measurement will provide the first precision measurement of the transverse asymmetry for the proton and deuteron. It will give us an opportunity to study the effects of twist-3 contributions to the structure functions. The kinematic region was chosen so that the data would connect closely with polarized DIS experiments, permitting direct comparison of extrapolated DIS data with our data and thus the study local duality for the polarized structure functions. Also, these data can be used to test the extended Gerasimov-Drell-Hearn [4] sum rule with a minimum of interpolations or use of fits to the world data on the structure function $g_{1}$. Along with other programs at Jefferson Lab [5] [6] [7], this measurement will contribute significantly to the world data on the spin structure functions.

\section{ACKNOWLEDGMENTS}

This work was supported by Department of Energy contract DE-FG02-96ER40950, and by the Institute of Nuclear and Particle Physics of the University of Virginia. The Southern Universities Research Association (SURA) operates the Thomas Jefferson National Accelerator Facility for the United States Department of Energy under contract DE-AC05-84ER40140.

\section{REFERENCES}

1. Abe, K., et al., Phys. Rev. Lett., 78, 815-819 (1997).

2. Adams, D., et al., Phys. Lett., B396, 338-348 (1997).

3. Ackerstaff, K., et al., Phys. Lett., B404, 383-389 (1997).

4. Burkert, V. D., and Ioffe, B. L., Phys. Lett., B296, 223-226 (1992).

5. Cates, G., and Meziani, Z.-E., Jefferson lab experiment 94-010.

6. Burkert, V., Crabb, D., and Minehart, R., Jefferson lab experiment 91-023.

7. Kuhn, S. E., Jefferson lab experiment 93-009. 